\title{
DISTRIBUCIÓN Y VARIABILIDAD DE LA PRECIPITACIÓN EN LA REGIÓN PAMPEANA, ARGENTINA
}

\author{
V.S. ALIAGA ${ }^{1 *}$, F. FERRELLI ${ }^{1}$, E.D. ALBERDI-ALGAÑARAZ ${ }^{1 \dagger}$, \\ V.Y. BOHN ${ }^{2}$, M.C. PICCOLO ${ }^{1,2}$ \\ Instituto Argentino de Oceanografía (IADO-CONICET), Buenos Aires, Argentina. \\ ${ }^{2}$ Departamento de Geografía y Turismo. Universidad Nacional del Sur (UNS), Buenos Aires, Argentina.
}

\begin{abstract}
RESUMEN. La Región Pampeana argentina es el principal centro de producción agropecuario del país y su economía depende, entre otros factores, del régimen de la precipitación. El objetivo del presente estudio fue zonificar esta región considerando la distribución espacio-temporal de la precipitación. Para ello, se aplicó un análisis de conglomerados sobre series mensuales de 33 estaciones meteorológicas pertenecientes al Servicio Meteorológico Nacional (SMN) durante el período 1960-2010. Como resultado se obtuvieron 6 subregiones. Los eventos extremos de precipitación en cada una de estas subregiones se estudiaron con el Índice Estandarizado de Precipitación (IEP) y la Transformada de Wavelet Continua (CWT). Se observó que la precipitación responde a variaciones interanuales y estacionales en distintos periodos e intensidad para cada subregión. Sin embargo, cuatro de ellas se caracterizaron por presentar homogeneidad pluviométrica, lo que permitió redefinir los límites de la región pampeana propuestos por Labraga et al. (2011). A partir de ahí se confeccionó una nueva regionalización pluviométrica teniendo en cuenta los patrones típicos de precipitación de la Región Pampeana.
\end{abstract}

\section{Distribution and variability of precipitation in the Pampas, Argentina}

ABSTRACT. Pampas region is the main agricultural area of Argentina. Its
economy mainly depends on rainfall regimes. The aim of this study was to
sub-regionalize the Pampas, considering the spatial and temporal distribution
of rainfalls. Cluster analysis was applied to study precipitation data from 33
meteorological stations from National Weather Service (SMN, Argentina)
during the period 1960-2010. As a result, 6 sub-regions were obtained.
Extreme precipitation events were studied with the Standardized Precipitation
Index (SPI) and Continuous Wavelet Transform (CWT). Rainfalls respond to
annual and seasonal timescales. Four of the sub-regions presented rainfalls
homogeneity, permitting to redefine the limits of the Pampas region proposed 
by Labraga et al. (2011). Thus, a new pluviometric regionalization was achieved considering the typical precipitation patterns of Pampas region.

Palabras clave: Región Pampeana, variabilidad de precipitaciones, regionalización, Argentina.

Key words: Pampas, precipitation variability, regionalization, Argentina.

Recibido 5 de octubre de 2015

Aceptado 16 de diciembre de 2015

*Correspondencia: V.S. Aliaga. Instituto Argentino de Oceanografía (IADOCONICET) - Florida 4500, B8000FWB, Bahía Blanca, Buenos Aires, Argentina. E-mail: valiaga@iado-conicet.gob.ar

\section{Introducción}

En áreas de llanura la dinámica de los recursos hídricos se relaciona directamente con la regularidad, intensidad y frecuencia de las precipitaciones. El conocimiento de eventos extremos de precipitación es relevante para orientar las políticas de ordenamiento del territorio referidas a obras de infraestructura y a diversas actividades económicas que deben ajustarse a las condiciones pluviométricas, como lo son la agricultura, ganadería y turismo (Zhao et al., 2012). La variabilidad de las precipitaciones afecta directamente a la capacidad de infiltración, la disponibilidad de nutrientes y carbono, suministro de servicios ecosistémicos, biomasa, productividad primaria, entre otros (Fay et al., 2011; Posada y Schuur, 2011; Yan et al., 2014).

En este trabajo el área de estudio corresponde a la Región Pampeana, Argentina definida por Labraga et al. (2011). Se trata de analizar en detalle las regiones fitogeográficas argentinas de la Pampa y el Espinal, que sufren extensos períodos de sequía o exceso de lluvia, que afectan a la disponibilidad de los recursos hídricos e impactan sobre la productividad de los sistemas agropecuarios y diversas actividades humanas. Ese autor se centró en el estudio de la predictibilidad y el desarrollo de métodos de pronóstico climático estacional de la lluvia en la Región Pampeana. La utilización de pronósticos climáticos eficientes permitiría prever el impacto de las fluctuaciones de la lluvia y planificar el manejo de los recursos hídricos (Labraga et al., 2011).

En el área de estudio la alternancia de períodos secos y húmedos y sus condiciones extremas tienen importantes consecuencias sociales y económicas. Algunas de las causas fueron la canalización de su drenaje como consecuencia de actividades agrícolas. Además, los procesos de eutrofización alteraron las comunidades de los ecosistemas lagunares (Scheffer, 1998). La existencia de ciclos naturales en la atmósfera afecta el régimen pluviométrico pampeano durante semanas, meses y décadas, con capacidad para alterar los ecosistemas. El ejemplo más destacado es El 
Niño Oscilación Sur (ENSO). Además, las oscilaciones interanuales de la precipitación se deben principalmente a la variabilidad en el otoño austral y el verano. Esta última es la temporada de máximas lluvias en la mayor parte del continente y de más influencia del ENSO en el cono sur de Sudamérica (Grimm, 2011).

El efecto de la variabilidad climática sobre la producción agropecuaria pampeana define la evolución de las variables económicas y los probables cambios en el uso de la tierra. Su impacto se expresa en la variación del rendimiento entre períodos pasados y escenarios climáticos futuros (Magrin et al., 2005). El área de estudio se ve afectada por periódicas inundaciones que afectan a las áreas rurales (sembrados, hacienda y viviendas) y zonas urbanas. Esto tiene importantes consecuencias económicas regionales (Taboada et al., 2009).

En los últimos 50 años se produjo en Argentina una sucesión de periodos de sequías meteorológicas severas e inundaciones significativas. Todas ellas afectaron a las actividades económicas, la dinámica de los cuerpos de agua y con ello la biodiversidad y las coberturas del suelo de la Región Pampeana (Barsky et al., 2008; Taboada et al., 2009). Forte Lay et al. (2008) establecieron las diferencias del régimen de lluvias entre los períodos 1947-1976 y 1977-2006, en los que se observó un incremento de la precipitación anual en el último período. Las diferencias de precipitaciones entre ambos ciclos superaron los $50 \mathrm{~mm}$ hasta alcanzar variaciones de 150 y $200 \mathrm{~mm}$. Las máximas amplitudes se registraron en el noreste de la provincia de Entre Ríos, mientras que variaciones de $150 \mathrm{~mm}$ se registraron en el centro de la región, la cual coincide con áreas donde estos cambios favorecieron el reemplazo de la ganadería por la agricultura.

Castañeda y Barros (1994), Barros et al. (2000) y Doyle et al. (2012) obtuvieron resultados similares en cuanto a la tendencia creciente de la precipitación a partir de 1960, principalmente en el extremo noreste de la región pampeana con algunas tendencias positivas de $5 \mathrm{~mm}$ por año y un máximo superior a $8 \mathrm{~mm}$ por año. El aumento de precipitaciones registrado en la Región Pampeana ocasionó notables incrementos en los rendimientos de los cultivos de secano (Magrin et al., 2005) y contribuyó de forma contundente a la expansión de las fronteras agrícolas y del área sembrada con cultivos anuales (Volante et al., 2015). Como evidencia de esto se observó un desplazamiento de la isohieta de $500 \mathrm{~mm}$ hacia el oeste durante la década de 1990, la cual constituye el límite occidental de la Región Pampeana, permitiendo la expansión de cultivos de grano (Sierra et al., 1994). Por lo tanto, es relevante actualizar la zonificación del área de estudio desde el punto de vista pluviométrico. Diversos autores han delimitado la Región Pampeana según temperatura y precipitación (Díaz y Mormeneo, 2002), según factores edáficos, aptitud de uso de la tierra y variables agro-climáticas (INTA-RIAP, Argentina), según características fitogeográficas, biodiversidad y servicios ambientales o recursos naturales y actividades económicas (Roccatagliata, 1988; Cabrera, 1994; Burkart et al., 1999; Bertonatti y Corcuera, 2000).

Teniendo en cuenta la heterogeneidad de las precipitaciones de la Región Pampeana, se realizó una clasificación en subregiones con el fin de caracterizar la 
variabilidad pluviométrica en distintas latitudes. El objetivo de este trabajo fue estudiar y caracterizar la alternancia de eventos pluviométricos en esta región durante el período 1960-2010 y generar una nueva delimitación del área de estudio que considere la distribución y variabilidad de la precipitación. La relevancia de este estudio es la de favorecer la predictibilidad y el desarrollo de métodos de pronóstico climático estacional de la precipitación de la Región Pampeana. Tales métodos, junto con los planes de ordenamiento del territorio, pueden mejorar no sólo la productividad agropecuaria sino también la calidad ambiental de la región con el fin de prevenir los efectos adversos de inundaciones, sequías, erosión, etc.

\section{Metodología}

La metodología propuesta se dividió en cuatro apartados. En el primero de ellos se describen los materiales utilizados. Los otros tres presentan los detalles de los métodos aplicados para subdividir la Región Pampeana y caracterizar el comportamiento pluviométrico de cada una de las subregiones obtenidas.

\subsection{Materiales}

Se consideró la Región Pampeana definida por Labraga et al., (2011) con una superficie de $613.532 \mathrm{~km}^{2}$ (Fig. 1). Se analizaron series mensuales pluviométricas correspondientes a 33 estaciones meteorológicas pertenecientes al Servicio Meteorológico Nacional (SMN, Argentina) para el período 1960-2010. Se obtuvieron 600 registros para cada estación, es decir que se estudiaron 19.800 datos en total. La calidad de los datos para la región de estudio fue probada por Díaz (2001) para el período 1961-1990. Con los registros de las dos décadas restantes se probó la normalidad, tendencia y consistencia teniendo en cuenta la guía de control de calidad de datos climatológicos de la Organización Meteorológica Mundial (OMM) en el Programa Mundial de Datos Climáticos (1984) aplicando la metodología expuesta por Rusticucci y Barrucand (2002).

\subsection{Método de Ward}

El objetivo de la agrupación es la nomenclatura estadística de objetos individuales a grupos o cluster. En este caso las estaciones meteorológicas fueron los objetos de agrupación sobre la base de datos pluviométricos mensuales para el período 19602010. Este procedimiento aglomerativo comienza su análisis con tantos grupos como individuos haya (estaciones meteorológicas, es decir, 33). A partir de estas unidades iniciales se van formando grupos de forma ascendente, hasta que al final del proceso todos los casos tratados están englobados en un mismo conglomerado o hasta que alcanza el número de grupos prefijados de acuerdo al grado de similitud deseado. Para este análisis se empleó el método jerárquico de Ward (1963) y un intervalo de distancia euclidiana al cuadrado como medida de disimilaridad. Esta última mide la distancia entre las estaciones. 

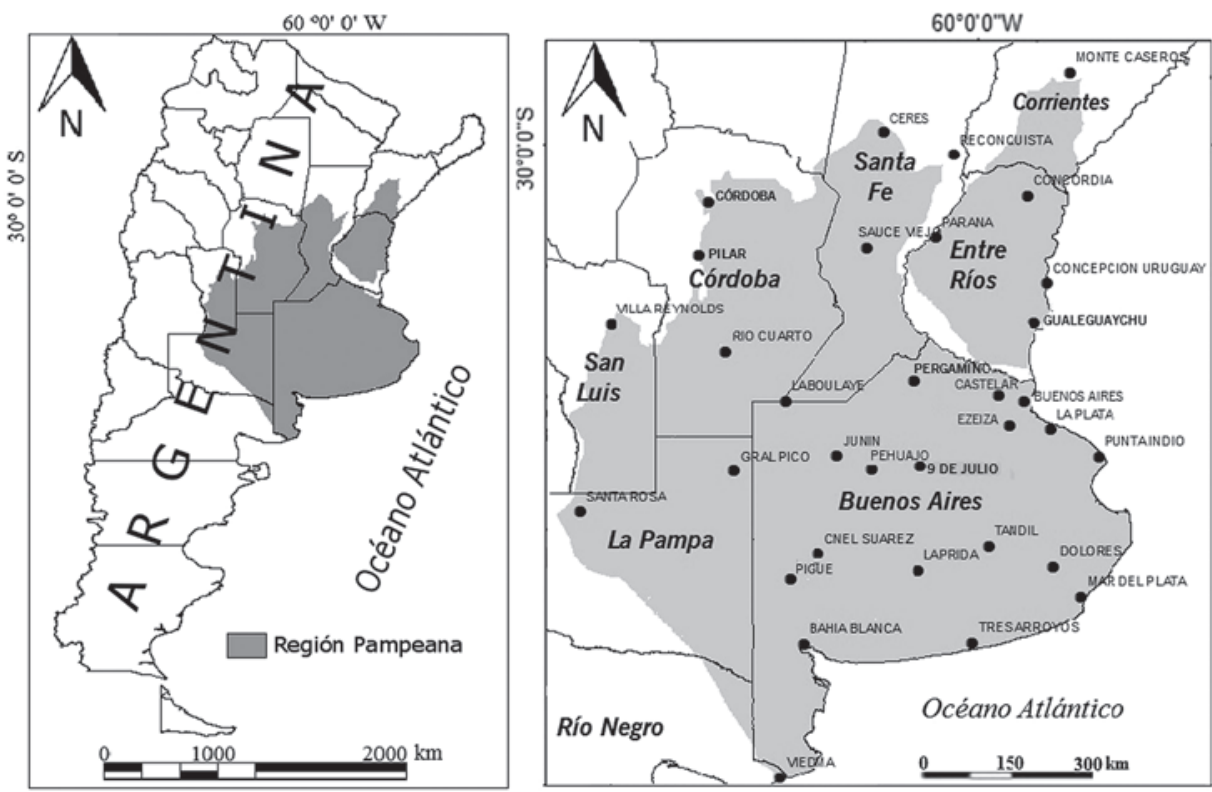

Figura 1. Región Pampeana (Argentina) y localización de las estaciones meteorológicas del SMN.

Este método agrupa por etapa y une los grupos de acuerdo al incremento en el valor total de la suma de las diferencias de cuadrados de cada grupo, de cada individuo al centroide de grupo. Donde $j$ es la variable, $i$ el individuo y $k$ cluster. $E_{k}$ es la suma de cuadrados de los errores del cluster $k$, o sea la distancia euclídea al cuadrado entre cada individuo del cluster $k$ a su centroide.

$$
E_{k}=\sum_{i=1}^{n k} \sum_{j=1}^{n}\left(x_{i j}^{k}-m_{j}^{k}\right)^{2}=\sum_{i=1}^{n k} \sum_{j=1}^{n}\left(x_{i j}^{k}\right)^{2}-n_{k} \sum_{j=1}^{n}\left(m_{j}^{k}\right)^{2}
$$

donde $E$ a la suma de cuadrados de los errores para todos los cluster, o sea, si suponemos que hay $h$ cluster.

$$
E=\sum_{k=1}^{h} E_{k}
$$

El método de agrupamiento aplicado en este trabajo se comparó con otros dos métodos jerárquicos, tales como el Vecino más lejano y Comparación de medias (Arthur, 2009). Sin embargo, el método de Ward fue el que mejor se ajustó al análisis de la precipitación. Este resultado coincide con lo estudiado por Fernau y Samson (1990), Gong y Richman (1995), Knapp et al. (2002) y Marzban y Sandgathe (2005). Este método agrupó las estaciones meteorológicas distribuidas en la región pampeana en seis grupos con diferencias o disimilitudes significativas menores al $2 \%$.

La representación espacial de esta zonificación se realizó a partir de la utilización de un Sistema de Información Geográfica (SIG) ArcGIS 9.3 a partir del método de interpolación Kriging Ordinario de tipo esférico. La estructura de correlación espacial entre los datos observados permite estimar la distribución espacial de precipitación (Hofstra 
et al., 2008; Ashiq et al., 2010). Este método es utilizado en particular para la representación mensual y anual de la precipitación (Goovaerts, 2000; Ninyerola et al., 2000; Diodato, 2005; Yang et al., 2011).

\section{3. Índice Estandarizado de Precipitación}

Tras la subregionalización del área de estudio, se calculó el Índice Estandarizado de Precipitación (IEP o SPI, por sus siglas en inglés Standardized Precipitation Index) sobre la precipitación media anual de cada subregión. La ventaja de este índice es que compara los déficits de precipitaciones en regiones con climas distintos y puede integrarse en un amplio margen de escalas temporales (Mckee et al., 1993). Para el cálculo del IEP en un lugar determinado se tiene en cuenta la serie histórica de precipitaciones mensuales correspondiente al período requerido. La misma es ajustada a la distribución teórica de probabilidad que se considere conveniente y se transforma en una distribución normal, de manera que el valor “0” se corresponde con los valores medios (Edwards y McKee, 1997).

El índice permite cuantificar las variaciones de las precipitaciones en distintas escalas temporales para a partir de ahí analizar el impacto del déficit de precipitación y sus efectos sobre los recursos hídricos. La categorización se realiza teniendo en cuenta los criterios presentados en la Tabla 1, basándose en la probabilidad acumulada según una distribución normal. La escala de análisis temporal fue de doce meses. Esta escala permite analizar las fluctuaciones que se generan desde un año hasta períodos más extensos. Este método permite un seguimiento de las precipitaciones y la determinación de períodos de sequías o inundaciones.

Tabla 1. Categorías del IEP modificado de Edwards y McKee (1997).

\begin{tabular}{|c|c|c|}
\hline Valores & Caracterización & Probabilidad Acumulada \\
\hline$>2$ & Extremadamente Húmedo & $0,9773-0,9986$ \\
\hline $1,5-1,99$ & Muy Húmedo & $0,9333-0,9772$ \\
\hline $1,0-1,49$ & Húmedo & $0,8414-0,9332$ \\
\hline $0,99--0,99$ & Casi Normal & $0,1588-0,8413$ \\
\hline$-1,0-1,49$ & Moderadamente Seco & $0,0669-0,1587$ \\
\hline$-1,49--1,99$ & Muy Seco & $0,0229-0,0668$ \\
\hline$<-2$ & Extremadamente seco & $0,0014-0,0228$ \\
\hline
\end{tabular}

\subsection{Transformada de Wavelet}

Con el objetivo de estudiar las series y los comportamientos temporales de la distribución de frecuencias de la precipitación en cada una de las subregiones discriminadas con el método de agrupamiento, se realizó un análisis de Transformada Wavelet Continua (Continous Wavelet Transform, CWT), que se define como (Mallat, 1999):

$$
(\widehat{W} S)(a, b)=|a|^{-\frac{1}{2}} \int S(t) \bar{\psi}_{a b} d t=\left\langle S, \bar{\psi}_{a b}>b \in \mathbb{R}, a>0\right.
$$

donde,

$$
\psi_{a b}=|a|^{-\frac{2}{2}} \psi\left(\frac{t-b}{a}\right)
$$


Esta función debe cumplir que $\lim _{t \rightarrow \pm \infty}|\psi|=0 \quad$ y que el valor medio sea nulo, p.e. $\int \psi(t) d t=0$ (Klees y Haagmans, 2000). El parámetro $a$ es un factor de escala de la función mother y el $b$ realiza una traslación en el tiempo. El valor de $(\hat{W} S)(a, b)$ es una medida de la correlación entre la mother y un segmento local de la señal analizada. Un valor alto indica que la señal tiene una mayor componente de la frecuencia correspondiente a la escala asignada. Con los parámetros anteriormente mencionados es posible reconstruir la variación temporal de la distribución de la amplitud de la señal en el espacio de frecuencia (Veltcheva y Soares, 2015). La wavelet utilizada fue la de Morlet, dado que ya ha sido aplicada con éxito para el estudio de la variabilidad de la precipitación (Coulibaly, 2006).

Para realizar este análisis se escribieron lineamientos (scripts) en el lenguaje Scilab (Software de acceso libre, www.scilab.org). El esquema aplicado fue el mencionado por Torrence y Compo (1998). Se analizaron las isolíneas que describieron los valores alcanzados por $|(\hat{W} S)(a, b)|$ y se obtuvo la distribución temporal y la periodicidad de los ciclos pluviométricos para cada subregión del área de estudio. Este método brinda una forma sencilla de analizar la descomposición de una serie de datos en tiempo-frecuencia distribuidas en el espacio. Permite además observar el modo dominante de variabilidad y la forma en que esos modos varían en el tiempo. Nakken (1999) utilizó esta técnica para identificar las componentes de la variabilidad en las precipitaciones y escorrentías derivadas del cambio climático y de factores antropogénicos. Además, este método permite estudiar, por ejemplo, la relación compleja entre la precipitación y las aguas subterráneas, tendencias entre caudal y precipitación y demás estudios climatológicos (Wang, 1995; Baliunas et al., 1997; Nalley et al., 2012; Yu y Lin, 2015).

\section{Resultados}

\subsection{Análisis de agrupamiento y su distribución espacial}

El análisis de agrupamiento permitió dividir las estaciones en conjuntos según su régimen de precipitaciones. Se seleccionaron 6 subregiones que mejor se ajustaron a la distribucion de la precipitación en la región pampeana. El árbol de asociación jerárquico se presenta en la Figura 2. Teniendo en cuenta el volumen medio anual de precipitaciones en el período de estudio (1960-2010), se le asignó a cada una un nombre representativo (Fig. 2): Extremadamente Húmeda (EH); Muy Húmeda (MH), Moderadamente Húmeda $(M o d H)$, Húmeda $(H)$, Seca $(S)$ y Muy Seca $(M S)$. La forma en que se agruparon permitió observar que los grupos $H$ y $S$ tuvieron la menores diferencias o mayor similitud entre ellos. Esto significa que la sub-región $H$ posee características pluviométricas similares a la $S$. De la misma manera, los grupos $E H$ y $M H$ presentaron características similares. Hacia el extremo árido, la $M S$ se relaciona más con el $H$ y $S$ que con la zona más húmeda, y el $M o d H$ fue más parecido a $E H$ y $M H$ (Fig. 2). 


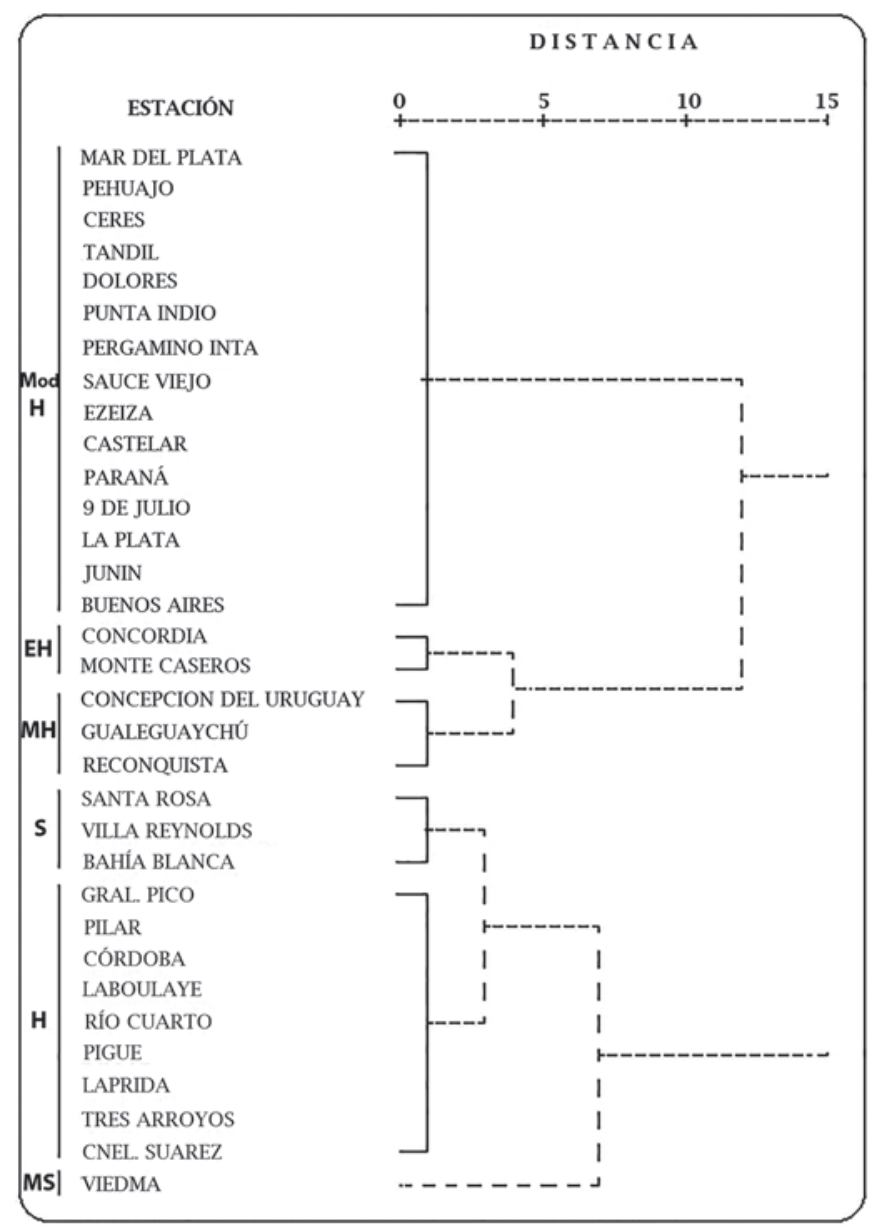

Figura 2. Dendograma de estaciones meteorologías en la región pampeana. De arriba abajo, Mod H: Moderadamente húmeda; EH: Extremadamente húmeda; MH: Muy húmeda; S: Seca; H: Húmeda; MS; Muy seca.

El curso anual de precipitación (Fig. 3) de cada grupo mostró una estacionalidad marcada de mayores lluvias durante los meses de otoño (marzo-abril) y primavera (octubre-noviembre). La estación seca se presentó en el invierno. Sin embargo, no en todos ellos se observó la misma amplitud anual. Los grupos más lluviosos presentaron mayores amplitudes anuales que los más secos, como son los casos de la subregión $H$ y la $M o d H$. Las mayores precipitaciones se concentraron en las subregiones $E H$, $M H, M o d H$, siendo el primero el que alcanzó las máximas precipitaciones mensuales (155 mm en el mes de abril). Naturalmente la subregión $M S$ presentó las menores precipitaciones en la región pampena con máximos medios mensuales de $45 \mathrm{~mm}$ y una amplitud anual de $23 \mathrm{~mm}$ (Fig. 3). La distribución espacial de estos seis grupos se presenta en la Figura 4. 


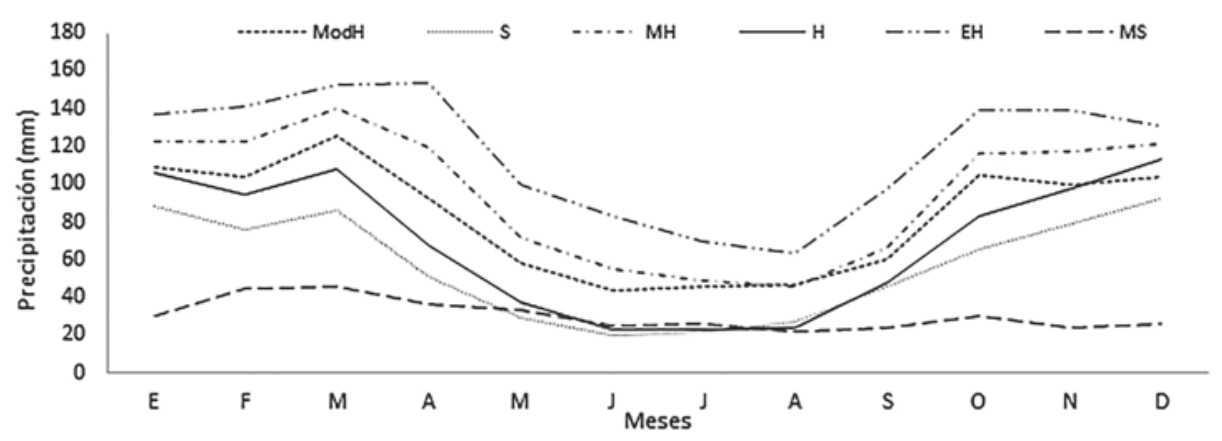

Figura 3. Régimen anual de precipitación para el período 1961-2010 en los seis grupos identificados.

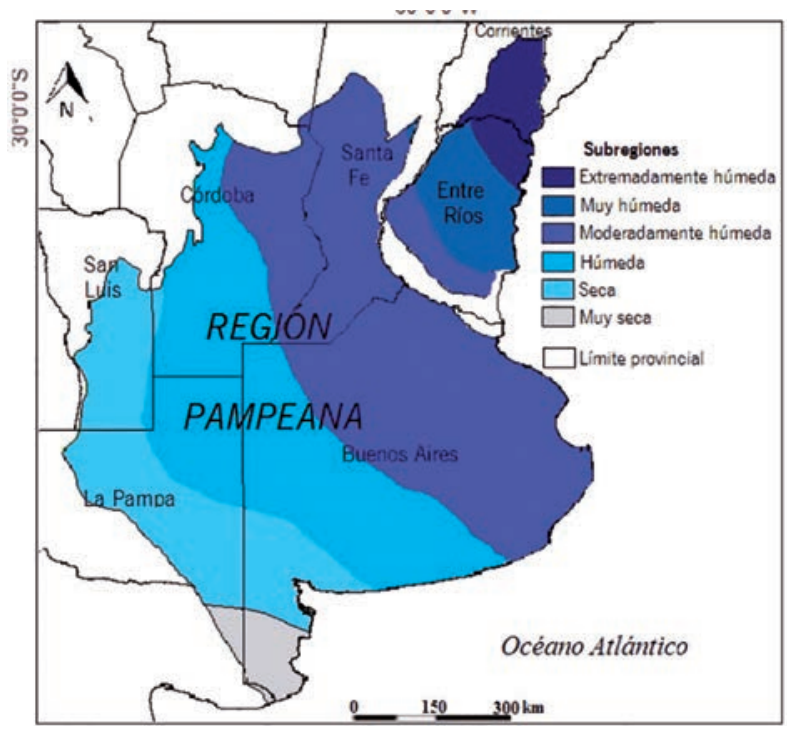

Figura 4. Mapa de zonificación de la región pampeana según su régimen pluviométrico.

Las mayores precipitaciones se concentraron en el extremo noreste de la Región Pampeana, representada por los grupos $E H$ y $M H$. El gradiente de precipitaciones disminuyó hacia el sudoeste como consecuencia de los efectos de la latitud. El promedio mensual de la precipitación en la subregión $M S$ es de aproximadamente $90 \mathrm{~mm}$ más baja que en las subregiones húmedas con medias mensuales de 80-120 mm. Las estaciones que se encuentran en el sudoeste de la Región Pampeana reflejaron la influencia de las condiciones secas nordpatagónicas.

\section{2. Índice Estandarizado de Precipitación (IEP)}

A partir de la definición de las subregiones, se aplicó el IEP para estudiar la precipitación media anual de cada una de ellas. Este índice permitió observar la ocurrencia de excesos o 
déficit significativos por su intensidad y/o duración, que no son homogéneos en cada subregión identificada (Fig. 5). La EH presentó las menores amplitudes del índice, por lo que los eventos extremos afectaron con menor intensidad el noreste de la región.

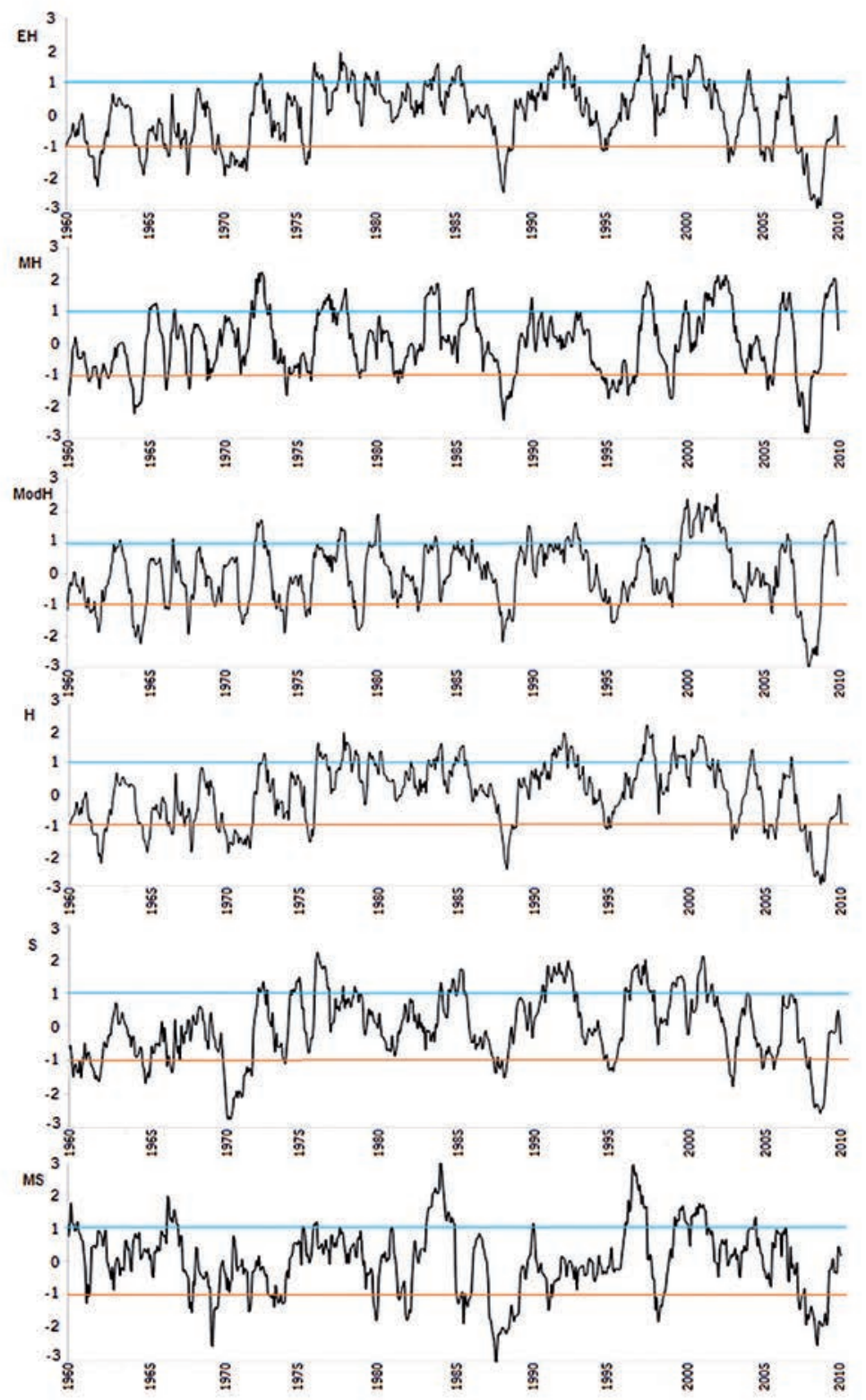

Figura 5. IEP a escala anual de la subregión Extremadamente húmeda (EH), Muy húmeda (MH), Moderadamente húmeda (ModH), Húmeda (H), Seca (S) y Muy seca (MS). 
Las subregiones $M H, M o d H$ y $H$ se ven afectadas simultáneamente por los eventos extremos de precipitación, tanto húmedos como secos en intensidad y duración. La subregión $S$ presentó una mayor cantidad de eventos secos que húmedos durante el período de estudio. $M S$ manifestó las condiciones de humedad y sequía más extremas, es decir, con las mayores amplitudes pluviométricas, sin embargo los períodos húmedos fueron de menor duración que los secos. $M H$ y $M o d H$ tuvieron amplitudes importantes en este índice, mientras que en el caso de la $E H$ manifestó los eventos húmedos y secos de menor intensidad (Fig. 5). A pesar de estas diferencias entre las subregiones, cabe destacar que los eventos Extremadamente Húmedos y Extremadamente Secos afectaron a toda la región pampeana. Por ejemplo, los eventos de extrema sequía registrados en los años 1988 y 2009 y los eventos muy lluviosos de 1997, 2000-2002 (Fig. 5).

\subsection{Transformada de Wavelet Continua}

Los resultados de CWT para la precipitación en cada subregión se presentan en la Figura 6. El eje $x$ representa el período de estudio en meses y años (50 años $=600$ meses). El eje $y$ indica el período de la frecuencia o señal en meses. Hacia la derecha se describe, en potencia, las señales predominantes para cada subregión (línea continua) mientas el cono de influencia (COI) se presenta con una línea punteada. Este último indica el área por encima de la cual los picos de mayor intensidad espectral no se ven afectados por los efectos de borde. Los que están por debajo son influenciados por los extremos de la muestra. La escala de colores a la derecha de cada caso representa magnitudes relativas de los coeficientes de CWT. Los máximos valores representan mayores relaciones entre el dato y la wavelet madre seleccionada. Los colores amarillo, naranja y rojo representan valores positivos de CWT y los azules indican correlación negativa (Hermida et al., 2015) (Fig. 6).

Se analizó el dominio de la componente anual en la señal de cada una de las subregiones. La señal anual fue más intensa en las subregiones centrales, mientras que en ambos extremos, representados por la subregión $E H$ y $M S$, fue menos predominante. Dentro de las subregiones de marcada señal anual, las subregiones $H$ y $S$ presentaron la mayor intensidad, mientras que la menor correspondió a $M H$ y $M o d H$. Las subregiones que se localizan en ambos extremos latitudinales también se destacaron por la presencia de eventos extremos de abundantes precipitaciones y sequías y por tener señales estacionales e interanuales más fuertes en comparación con las restantes.

Con respecto a las frecuencias interanuales y estacionales, en la subregión $E H$ se destacaron señales periódicas de tres, cinco y seis meses, las cuales podrían estar relacionadas con la Oscilación Madden Julian (MJO) que posee un período de 60 a 90 días. Se observaron también señales estacionales de 6 meses en las subregiones $M H$ y $M o d H$ que coincidieron con las estaciones lluviosas de otoño y primavera (Fig. 3).

En el caso de las señales interanuales se observaron ciclos de cinco años de notable intensidad en las dos subregiones más húmedas $(E H$ y $M H)$ y de 3 años en la $E H$ (Fig. 6.a). En la $M H$ el ciclo de 3 años identificó los eventos de mayor duración y la de 5 años se presentó en todo el período de estudio (Figura 6.b). Por el contrario, los eventos de mayor duración (8 a 10 años) se observaron en la subregión $M S$ (Figura 6.f). Una de las principales fuentes de variabilidad de las precipitaciones a escala interanual en la Región 

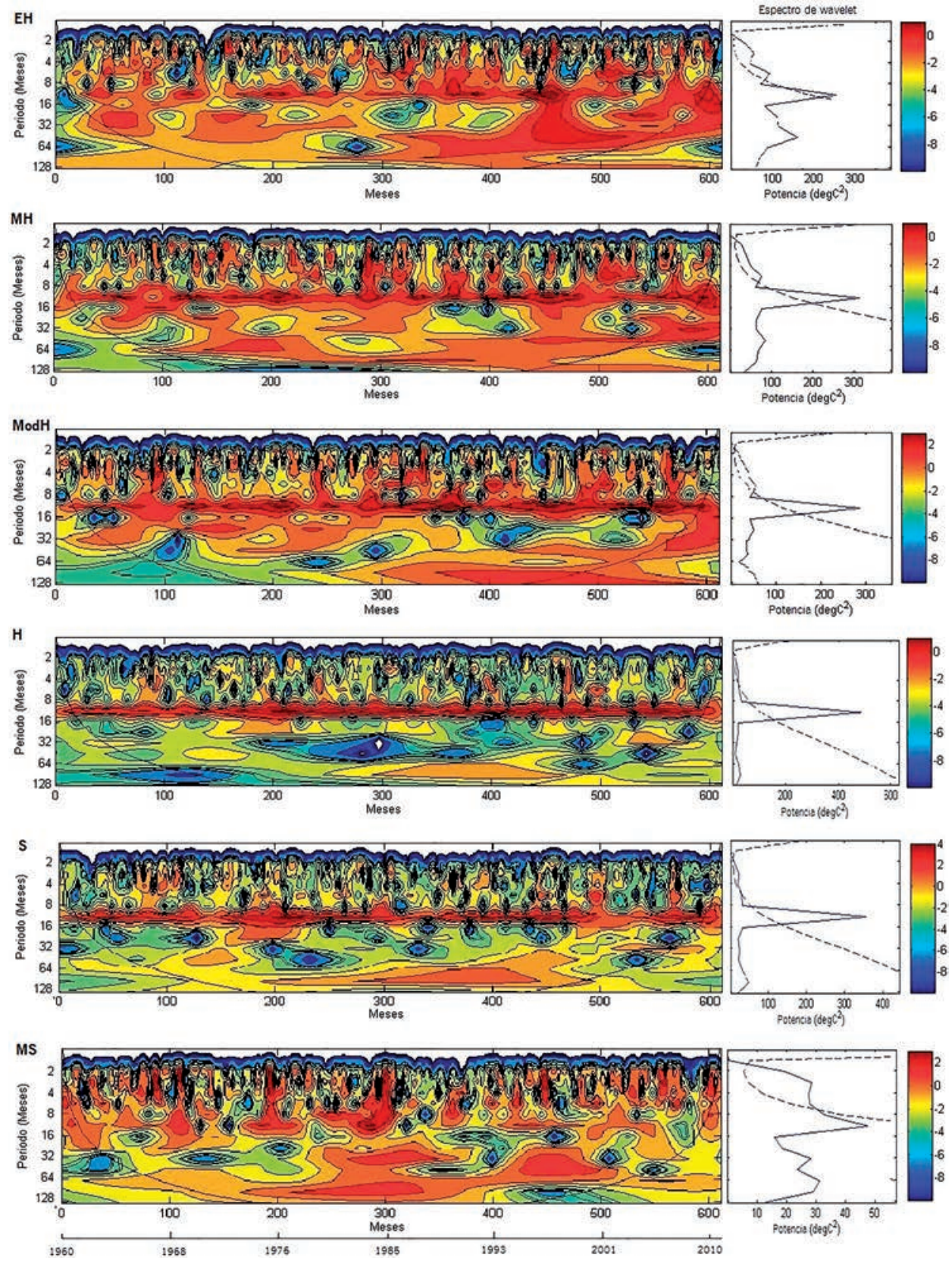

Figura 6. CWT de precipitación en la subregión Extremadamente húmeda (EH), Muy húmeda $(M H)$, Moderadamente húmeda (ModH), Húmeda (H), Seca (S) y Muy seca (MS). 
Pampeana es el ENSO (El Niño Oscilación Sur), cuyas fases extremas son conocidas como eventos El Niño (EN) y La Niña (LN). La subregión $M H$ evidenció períodos de elevadas precipitaciones durante los años 1991-92 y 2002 que coinciden con eventos moderados EN según indica el Índice Oceánico de El Niño (ONI) desarrollado por la National Oceanic and Atmospheric Administration (NOAA) (http://www.cpc.ncep.noaa.gov/). Por otro lado, en la subregión $M o d H$ también se registraron períodos muy lluviosos durante los mismos años pero de mayor intensidad y duración.

En la subregión $H$ la componente de 4 años marcó ciclos lluviosos, destacando el período 1980-1987, de los cuales los años 1980-1982 se caracterizaron como EN muy fuertes mientras que en 1983-1987 fueron moderados. En el caso de la subregión $S$, se observaron periodos húmedos de variación estacional e interanual. Sin embargo, los eventos más intensos fueron los secos con una señal de 8 años. Por último, la $M S$ tuvo componentes de 3 y 4 años de períodos lluviosos aunque los secos son de mayor intensidad y duración. Teniendo en cuenta la distribución y comportamiento de la precipitación durante el período de estudio, puede afirmarse que las subregiones $M H, M o d H, H$ y $S$ presentaron homogeneidad, que responde principalmente a su comportamiento anual. Las subregiones $E H$ y $M S$ tuvieron respuestas diferentes, seguramente al estar influenciadas por regímenes pluviométricos que exceden a las características típicas de la Región Pampeana. En el caso de la $E H$, por la influencia subtropical y en la $M S$, por el dominio nordpatagónico.

\section{Discusión}

En la literatura hay numerosos trabajos que analizan la precipitación y delimitan regiones basándose en el método de agrupamiento de Ward. Entre ellos destacan los estudios realizados en Perú, (Laura et al., 2015), México (Ramos Soto, 2011), Colombia (Jaramillo y Chaves, 2000), Estados Unidos (Baeriswyl y Rebetez, 1997), Irán (Modarres, 2006) y Suiza (Marzban y Sandgathe, 2005). En ellos se utilizaron registros mensuales o diarios de precipitación. La aplicación de este método permitió analizar la distribución espacial y el comportamiento temporal de las precipitaciones en la Región Pampeana. Su extensión la vuelve compleja y heterogénea. El análisis de wavelet permitió analizar las diferencias de frecuencia y periodicidad de los eventos extremos húmedos y secos. Siguiendo el lineamiento de estudio realizado por Díaz y Mormeneo (2002), se trabajó con seis grupos en lugar de cuatro y se extendió el período de estudio a 50 años. Además se clasificó toda la Región Pampeana. Esto permitió analizar la variabilidad de las precipitaciones e identificar eventos extremos comparando su intensidad y periodicidad en cada subregión.

En diversos estudios se ha caracterizado a la Región Pampeana según factores edáficos, aptitud de uso de la tierra, y variables agro-climáticas, y los límites de las mismas obedecen a los límites político-administrativos (INTA-RIAP, Argentina). Existen además delimitaciones que se basan en la biogeografía definiendo eco-regiones según sus características fitogeográficas, biodiversidad y servicios ambientales o recursos naturales y actividades económicas, entre otras (Roccatagliata, 1988; Cabrera, 1994; Burkart et al., 1999; Bertonatti y Corcuera, 2000). Sin embargo, se carece de una delimitación netamente pluviométrica. 
La variabilidad de las precipitaciones de la Región Pampeana afecta a los suelos, la disponibilidad de nutrientes y la actividad primaria. Las sequías e inundaciones afectan de forma particular a una parte de la región, pero la extensión del evento es impredecible (Márdero et al., 2012). Por otro lado, durante los eventos secos se ha observado una disminución de las áreas cultivadas y las destinadas a pastos para el ganado, promoviendo procesos de erosión eólica, salinización de los suelos, mortandad de animales, etc. (Michelena et al., 2008; Prieto y Herrera, 2001; Quirós et al., 2002; Maizels et al., 2003, Geraldes y Boavida, 2005; Scarpati y Capriolo, 2013). Durante los eventos de extrema sequía registrados en los años 1988 y 2009 y los eventos muy lluviosos de 1997 y 20002002 se observaron consecuencias socioeconómicas significativas como la reducción notable de la cobertura de cultivos y pastos al aumentar la superficie ocupada por suelos al descubierto (Ferrelli et al., 2011; Ferrelli, 2012). Además, los efectos de este fenómeno se observan en la reducción de la profundidad de las lagunas y la escasez de peces (Quirós et al., 2002). En contraposición, el aumento de las precipitaciones generó una extensión del área cultivada, un aumento del agua potable para los habitantes y un incremento de las superficie lagunar (Sosnovsky y Quirós, 2006; Ferrelli, 2012).

Las frecuencias interanuales y estacionales identificadas en la subregión $E H$ de tres, cinco y seis meses podrían estar relacionadas con la Oscilación Madden Julian (MJO) que posee un período de 60 a 90 días (Bridgman y Oliver, 2006). Esta oscilación de la atmósfera tropical-oceánica afecta a la variabilidad de las precipitaciones, principalmente en latitudes bajas, coincidiendo con el extremo noreste de la Región Pampeana influenciado por el anticiclón del Atlántico Sur. Según González (2010) la actividad de la MJO se desarrolla a través de trenes de onda de Rossby que conectan el Continente Marítimo con Sudamérica.

Los resultados obtenidos coinciden con lo estudiado por Barros et al. (2000): las tendencias de precipitación anual positivas pueden atribuirse en parte a los cambios en la frecuencia e intensidad de las fases del ENSO. La ocurrencia de lluvias intensas se da en períodos cortos de tiempo pero con mayor frecuencia, especialmente entre los 30 y $40^{\circ} \mathrm{S}$ de latitud (Barros et al., 2000). Grimm (2011) evidenció que el ENSO ejerce una influencia más amplia y significativa en la frecuencia de eventos extremos de precipitación que en las anomalías mensual o estacional. Esto aumenta los riesgos ante eventos intensos de corta duración con consecuencias más drásticas. Se observó que en la Región Pampeana la contribución más importante a la variación anual de la precipitación proviene de la variabilidad en el otoño y en menor medida en el verano.

Finalmente, teniendo en cuenta el análisis de la distribución de la precipitación y la comparación de las características subregionales de la Región Pampeana, fue posible establecer nuevos límites. Estos representan un área con características pluviométricas típicas de una región templada húmeda y subhúmeda y responden únicamente a las condiciones pluviométricas. De esta manera, desde un punto de vista pluviométrico, la Región Pampeana se define por el área delimitada por las subregiones $M H, M o d H, H$ y $S$ (Fig. 7). El conocimiento de estos límites permitirá caracterizar y comprender otros aspectos sociales y económicos de la región, así como prevenir efectos adversos sobre la población y sus actividades económicas. Se demostró que la sub-región $M S$ presenta un comportamiento norpatagónico caracterizado por la presencia de una masa de aire 
con muy poca humedad (Soriano, 1956), mientras que la $E H$ se caracteriza por un comportamiento subtropical. En ninguno de estos casos se mantuvo una clara relación con el régimen pampeano, por lo que se las excluyó de la delimitación.

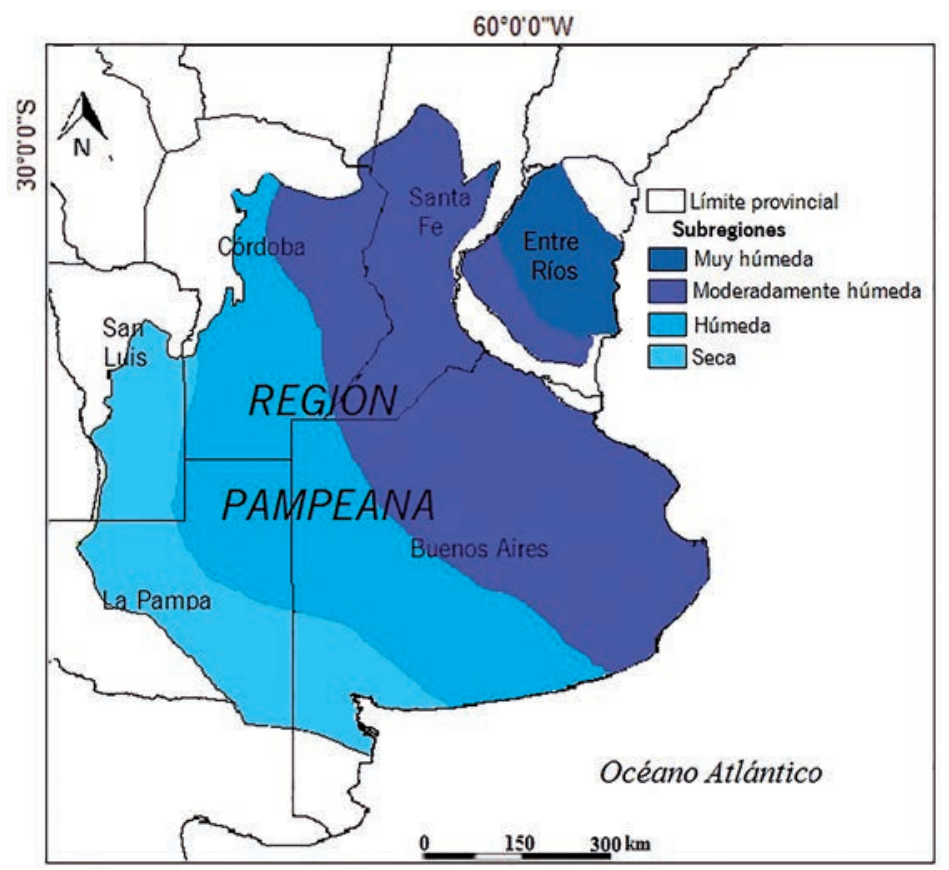

Figura 7. Nueva delimitación de la Región Pampeana según su régimen pluviométrico para el período 1960-2010.

La región sudoeste de la Provincia de Buenos Aires que coincide con la $M S$ se encuentra por debajo de la isohieta de $500 \mathrm{~mm}$. No obstante, existen ciclos climáticos en que esos valores se modifican. Su ecosistema natural se ha mantenido en períodos secos gracias a la cobertura del suelo con especies arbóreas, arbustivas y pastizales que han permitido conservar la humedad y evitar la degradación en una zona donde prevalecen fuertes vientos que favorecen los procesos de erosión. Sin embargo, el avance de la actividad agrícola a expensas de la ganadería y la eliminación de la cobertura natural arbórea y arbustiva ha incrementado la superficie ocupada por tierras degradadas hasta llegar en la actualidad a procesos de desertificación en extensas áreas. La consecuencia directa de este proceso es la disminución progresiva de la producción agropecuaria, mientras los ecosistemas se ven cada vez más afectados en cuanto a su potencial de respuesta ante los impactos negativos del cambio climático y su capacidad de recuperación (OPDS, 2012). En el otro extremo, el área comprendida por la subregión $E H$ presenta condiciones subtropicales. La práctica de la ganadería resulta difícil por las altas temperaturas y el predominio de pastos relativamente pobres, y su agricultura se centra en el desarrollo de cultivos de arroz y yerba mate (Alarcón, 2013). Las condiciones medioambientales de ambas subregiones ( $M S$ y $E H$ ) 
sumadas a la variabilidad de las precipitaciones, exigen que las técnicas de producción agropecuaria deban adecuarse a las limitaciones del medio.

\section{Conclusiones}

En este trabajo se demostró que la variabilidad de la precipitación en la Región Pampeana es muy elevada. La aplicación del método de conglomerados permitió dividir la región de estudio en seis subregiones cuya nomenclatura se relacionó con el volumen de precipitación, distinguiéndose cuatro regiones húmedas en las que las precipitaciones anuales fueron superiores a los $650 \mathrm{~mm}$ y dos regiones secas. La zonificación fue útil para analizar la variabilidad de las precipitaciones, su gradiente y la duración e intensidad de los eventos extremos.

Se observó que los eventos extremos fueron más intensos a medida que aumentó la aridez del ambiente. En la subregión Muy Seca, se observaron menor cantidad de eventos extremos pero de mayor intensidad. En el caso opuesto, la región Extremadamente Húmeda presentó una mayor cantidad de eventos extremos pero la mayoría de ellos no superó la escala de moderadamente húmedo o moderadamente seco según el IEP. Los eventos extremos de mayor intensidad afectaron a toda la Región Pampeana, mientras que los de menor intensidad fueron observados sólo en algunas subregiones. Ejemplo de ello son los años 2002 y 2008 caracterizados por abundantes lluvias y sequías severas, respectivamente. La subregionalización permitió captar diferencias en eventos débiles (según ONI). El análisis de las precipitaciones en relación al ONI demostró que los eventos El Niño y La Niña tienen gran influencia en el área de estudio, observándose además que el impacto de estos fenómenos varía de una subregión a otra.

Se propuso una nueva delimitación de la región pampeana que considera la distribución de la precipitación durante el periodo 1960-2010 (Fig. 7). Este conocimiento constituye un aporte importante para la gestión territorial, ya que el estudio de los distintos regímenes pluviométricos ayuda a la planificación de los calendarios agrícolas y a la gestión del territorio. La disponibilidad de recursos hídricos marca el desarrollo de numerosas actividades agroeconómicas dependientes de las precipitaciones in situ. La caracterización de los regímenes pluviométricos a escala subregional es una herramienta necesaria para generar acciones de prevención de procesos como la erosión, las sequías y las inundaciones que podrían impactar sobre la productividad.

\section{Referencias}

Alarcón, M.F. 2013. Aproximación al análisis de la capacidad de acogida del territorio para el desarrollo de la actividad agrícola: el caso de la cuenca del río Miriñay, provincia de Corrientes (Argentina). XIV Encuentro de Geógrafos de América Latina (EGAL-2013), Unión Geográfica Internacional - Comité Nacional de Perú, Lima, 18 pp.

Arthur, D. 2009. Analyzing and improving local search: k-means and ICP. Stanford University. Computer Science Dept., 228 pp.

Ashiq, M., Zhao, C., Ni, J., Akhtar, M. 2010. GIS-based high-resolution spatial interpolation of precipitation in mountain-plain areas of Upper Pakistan for regional climate change impact studies. Theoretical and Applied Climatology 99 (3), 239-253. 
Baeriswyl, P.A., Rebetez, M. 1997. Regionalization of Precipitation in Switzerland by Means of Principal Component Analysis. Theoretical and Applied Climatology 58, 31-41.

Baliunas, S., Frick, P., Sokoloff, V., Soon, V. 1997. Time scales and trends in the central England temperature data (1659-1990): A wavelet analysis. Geophysical Research Letters 24, 13511354.

Barros, V.R., Castañeda, M.E., Doyle, M.E. 2000. Recent precipitation trends in Southern South America East of the Andes: an indication of climatic variability. In P.P. Smolka, V. Volkheimer, (eds.), Southern Hemisphere Paleo and Neoclimates: Key Sites, Methods, Data and Models. Berlin, Springer-Verlag, pp. 187-206.

Bertonatti, C., Corcuera, J. 2000. Situación ambiental Argentina 2000. Buenos Aires, Fundación Vida Silvestre, $391 \mathrm{pp}$.

Burkart, R., Bárbaro, N.O., Sánchez, R.O., Gómez, D.A. 1999. Eco-regiones de la Argentina. Programa Desarrollo Institucional Ambiental, Secretaría de Recursos Naturales y Desarrollo Sustentable, Administración de Parques Nacionales, Buenos Aires, 42 pp.

Bridgman, H.A., Oliver, J.E. 2006. The global climate system: patterns, processes, and teleconnections. Cambridge University Press, Cambridge.

Cabrera, A.L. 1994. Enciclopedia Argentina de agricultura y jardinería Tomo II, Fascículo 1: regiones fitogeográficas Argentinas. ACME, Buenos Aires.

Castañeda, M.E., Barros, V.R. 1994. Las tendencias de la precipitación en el Cono sur de América al Este de los Andes. Meteorológica 19, 23-32.

Climate Prediction Center. 2015. Oceanic Niño Index (ONI). Disponible en: http://www.cpc.ncep. noaa.gov/data/indices/oni.ascii (fecha de acceso: 20/03/2015).

Coulibaly, P. 2006. Spatial and temporal variability of Canadian seasonal precipitation (19002000). Advances in Water Resources 29, 1846-1865.

Díaz, R.A. 2001. Calidad de las series mensuales de temperaturas y lluvias en Argentina. Actas de la III Reuniao Latino-Americana de Agrometeorología, Fortaleza, Brasil, pp. 641-642.

Díaz, R.A., Mormeneo, I. 2002. Zonificación del clima de la región Pampeana mediante análisis de conglomerados con consenso. Revista Argentina de Agrometeorología 2 (2), 125-131.

Diodato, N. 2005. The influence of topographic co-variables on the spatial variability of precipitation over small regions of complex terrain. International Journal of Climatology 25 (3), 351-363.

Doyle, M., Saurral, R., Barros, V.R. 2012. Trends in the distributions of aggregated monthly precipitation over the Plata Basin. International Journal of Climatology 32, 2149-2162.

Edwards, D., Mckee, T. 1997. Characteristics of 20th century drought in the United States at multiple time scales. Climatology Report 97-2, Colorado State University.

Fay, P.A., Blair, J.M., Smith, M.D., Nippert, J.B., Carlisle, J.D., Knapp, A.K. 2011. Relative effects of precipitation variability and warming on tall grass prairie ecosystem function. Biogeosciences 8, 3053-3068.

Fernau, M.E., Samson, P.J. 1990. Use of cluster analysis to define periods of similar meteorology and precipitation chemistry in eastern North America. Part I: Transport patterns. Journal of Applied Meteorology 29 (8), 735-750.

Ferrelli, F. 2012. La sequía 2008-2009 en el sudoeste de la provincia de Buenos Aires (Argentina). Ecosistemas 21, 235-238.

Ferrelli, F., Bohn, V.Y., Piccolo M.C. 2011. Aplicación de geotecnologías al estudio de las precipitaciones y su relación con las coberturas del suelo (Pcia. de Buenos Aires, Argentina). GeoFocus Revista Internacional de Ciencia y Tecnología de la Información Geográfica 11, 355-374.

Forte Lay, J., Scarpati, O., Capriolo, A. 2008. Precipitation variability and soil water content in Pampean Flatlands (Argentina). Geofísica Internacional 47 (4), 341-354. 
Geraldes, A.M., Boavida, M.J.L. 2005. Seasonal water level fluctuations: implications for reservoir limnology and management. Lakes \& Reservoires: Research and Management 10, 59-69.

Gong X., Richman M.B. 1995. On the application of cluster analysis to growing season in precipitation data in North America East of the Rockies. Journal of Climate 8, 897-931.

González, P.L.M. 2010. Dinámica asociada a la variabilidad intraestacional de la precipitación de verano en Sudamérica. Tesis Doctoral, Facultad de Ciencias Exactas y Naturales. Universidad de Buenos Aires, 288 pp. Disponible en: http://digital.bl.fcen.uba.ar/Download/ Tesis/Tesis_4674_Gonzalez.pdf.

Goovaerts, P., 2000. Geostatistical approaches for incorporating elevation into the spatial interpolation of rainfall. Journal of Hydrology 228 (1-2), 113-129.

Grimm, A.M. 2011. Interannual climate variability in South America: impacts on seasonal precipitation, extreme events, and possible effects of climate change. Stoch. Environ. Res. Risk Assess. 25, 537-554.

Hermida, L., López, L., Merino, A., Berthet, C., García-Ortega, E., Sánchez, J.L., Dessens, J. 2015. Hailfall in southwest France: Relationship with precipitation, trends and wavelet analysis. Atmospheric Research 156, 174-188.

Hofstra, N., Haylock, M., New, M., Jones, P., Frei, C. 2008. Comparison of six methods for the interpolation of daily European climate data. Journal of Geophysical Research 113, D21110.

Jaramillo, R.A., Chaves, C.B. 2000. Distribución de la precipitación en Colombia analizada mediante conglomeración estadística. Cenicafé 51 (2), 102-113.

Klees R., Haagmans, R. 2000. Wavelets in the Geosciences. Springer-Verlag, Berlin, 241 pp.

Knapp, P.A, Grissino-Mayer, H.D., Soulé, P.T. 2002. Climatic Regionalization and the SpatioTemporal Occurrence of Extreme Single-Year Drought Events (1500-1998) in the Interior Pacific Northwest, USA. Quaternary Research 58, 226-233.

Labraga, J., Brandizi, L., López, M. 2011. Avances en el pronóstico climático de las anomalías de lluvia en la Región Pampeana. Meteorológica, 36 (2), 59-71.

Laura, E.J., Obando, O.G.F. 2015. Análisis de frecuencia regional de las precipitaciones máximas diarias en la región hidrográfica del Titicaca. Revista de Investigación Altoandina 17 (1), 53-64.

Maizels, P., Etchepare, E., Chornomaz, E., Bustingorry, J., Escaray, R., Conzonno, V. 2003. Parámetros abióticos y biomasa planctónica en la laguna Chascomús (Pcia. de Buenos Aires). Período de inundación 2002. Biología Acuática 20, 6-11.

Magrin, G.O., Travasso, M.I., Rodríguez, G.R. 2005. Changes in climate and crop production during the 20th century in Argentina. Climatic Change 72, 229-249

Mallat, S. 1999. A Wavelet tour of Signal Processing. Wavelet Analysis \& Its Applications. Academic Press, San Diego, 637 pp.

Márdero, S., Nick1, E., Schmook, B., Schneider, L., Rogan, J., Christman, Z., Lawrence, D. 2012. Sequías en el sur de la península de Yucatán: análisis de la variabilidad anual y estacional de la precipitación. Investigaciones Geográficas 78, 19-33.

Marzban, C., Sandgathe, S. 2005. Cluster analysis for verification of precipitation fields. Weather and Forecasting 21, 824-838.

Mckee, T., Doeksen, N.J., Kliest, J. 1993. The relationship of drought frequency and duration to times scales. California, EE.UU. Preprint 8 th Conference on Applied Climatology 17-22, 179-184.

Michelena, R., Morrás, H., Irurtia, C. 1996. Degradación física por agricultura continua de suelos franco-limosos de la provincia de Córdoba. Congreso Argentino de la Ciencia del Suelo, Santa Rosa de La Pampa, Argentina. 
Modarres, R. 2006. Regional Precipitation Climates of Iran. Journal of Hydrology (NZ) 45 (1), 13-27.

Nakken, M. 1999. Wavelet analysis of rainfall-runoff variability isolating climatic from anthropogenic patterns. Environmental Modelling \& Software 14, 283-295.

Nalley, D., Adamowski, J., Khalil, B. 2012. Using discrete wavelet transforms to analyze trends in streamflow and precipitation in Quebec and Ontario (1954-2008). Journal of Hydrology 475, 204-228.

Ninyerola, M., Pons, X., Roure, V. 2000. A methodological approach of climatological modelling of air temperature and precipitation through GIS techniques. International Journal of Climatology 20 (14), 1823-1841.

OPDS: Organismo Provincial para el Desarrollo Sostenible. 2012. Proyecto "Incremento de la Resiliencia Climática y Mejora de la Gestión Sustentable del Suelo en el Sudoeste de la Provincia de Buenos Aires". Secretaria de Medio Ambiente y desarrollo sustentable de la Nación. Análisis y evaluación social, Anexo V. Disponible en: http://www.ambiente.gov.ar/archivos/ web/DCSyLD/file/Joaquin/Evaluacion\%20Social\%20Anexo\%20V.pdf

Posada, J.M., Schuur, E.A.G. 2011. Relationships among precipitation regime, nutrient availability, and carbon turnover in tropical rain forests. Oecologia 165 (3), 783-795.

Prieto, M.D.R., Herrera, R.G. 2001. De sequías, hambrunas, plagas y otras varias y continuas calamidades acaecidas en la jurisdicción de Córdoba durante el siglo XVIII. Cuadernos de Historia. Serie Economía y Sociedad 4, 131-158.

Quirós, R., Rennella, A., Boveri, M., Rosso, J., Sosnovsky, A. 2002. Factores que afectan la estructura y el funcionamiento de las lagunas pampeanas. Ecología Austral 12, 175-185.

Ramos Soto, J.C. 2011. Determinación de eventos de precipitación significativa usando análisis de conglomerados (cluster análisis) para México durante los últimos 40 años. Instituto Mexicano de Tecnología del Agua, 128 pp.

Roccatagliata, J.A. 1988. La Argentina: Geografía general y los marcos regionales. Buenos Aires, Planeta, 783 pp.

Rusticucci, M.M., Barrucand, M.G. 2002. Climatología sobre temperaturas extremas en la Argentina. Consistencia de datos. Relación entre la temperatura media estacional y la ocurrencia de días extremos. Meteorológica 26 (1-2), 65-79.

Scheffer, M. 1998. Ecology of shallow lakes. Population and Community Biology Series. Kluwer Academic Publishers, Dordrecht, 358 pp.

Sierra, E.M., Hurtado, H.R., Specha, L. 1994. Corrimiento de las isohietas anuales medias decenales en la Región Pampeana 1941-1990. Rev. Facultad Agronomía 14 (2), 139-144.

Scarpati, O.E., Capriolo, A.D. 2013. Sequías e inundaciones en la provincia de Buenos Aires (Argentina) y su distribución espacio-temporal. Investigaciones Geográficas 82, 38-51.

Soriano, A. 1956. Los distritos norísticos de la provincia Patagónica. Revista de Investigaciones Agropecuarias 10, 323-347.

Sosnovsky, A., Quirós, R. 2006. El estado trófico de pequeñas lagunas pampeanas, su relación con la hidrografía y el uso de la tierra. Ecología Austral 16, 115-124.

Taboada, M.A., Damiano, F., Lavado, R.S. 2009. Inundaciones en la Región Pampeana. Consecuencias sobre los suelos. Alteraciones de la fertilidad de los suelos: el halomorfismo, la acidez, el hidromorfismo y las inundaciones. Universidad de Buenos Aires, Buenos Aires, pp. 103-127.

Torrence, C., Compo, G.P. 1998. A practical guide to wavelet analysis. Bulletin of the American Meteorological Society 79, 61-78.

Veltcheva, A., Guedes Soares, C. 2015. Wavelets analysis of non-stationary sea waves during Hurricane Camille. Ocean Engineering 95, 166-174. 
Volante, J., Mosciaro, J., Morales Poclava, M., Vale, L., Castrillo, S., Sawchik, J., Paruelo, J. 2015. Expansión agrícola en Argentina, Bolivia, Paraguay, Uruguay y Chile entre 2000-2010: Caracterización espacial mediante series temporales de índices de vegetación. RIA. Revista de Investigaciones Agropecuarias 41 (2), 179-191.

Ward, J.H. 1963. Hierarchical grouping to optimize an objective function. Journal American Statistical Association 58, 236-244.

Wang, B. 1995. Interdecadal changes in El Niño onset in the last four decades. Journal of Climate $8,267-285$.

Weng, H., Lau, K.M. 1994. Wavelets, period doubling, and time-frequency localization with application to organization of convection over the tropical western Pacific. Journal of the Atmospheric Sciences 51, 2523-2541.

Yan, L., Chen, S., Xia, J., Lou, Y. 2014. Precipitation regime shift enhanced the rain pulse effect on soil respiration in a semi-arid steppe. PLoS ONE 9 (8) e104217. Doi: 10.1371/journal. pone.0104217.

Yang, H., Li, Y., Wu, M., Zhang, Z., Li, L.Wan, S. 2011. Plant community responses to nitrogen addition and increased precipitation: the importance of water availability and species traits. Global Change Biology 17, 2936-2944.

Yu, H.L., Lin, Y.C. 2015. Analysis of space-time non-stationary patterns of rainfall-groundwater interactions by integrating empirical orthogonal function and cross wavelet transform methods. Journal of Hydrology 525, 585-597.

Zhao, Q., Liu, S., Deng, L., Dong, S., Yang, J., Wang, C. 2012. The effects of dam construction and precipitation variability on hydrologic alteration in the Lancang River Basin of southwest China. Stochastic Environmental Research and Risk Assessment 26, 993-1011. 